Pacific

Journal of

Mathematics

A DE RHAM THEOREM FOR SYMPLECTIC QUOTIENTS

REYER SJAMAAR 


\title{
A DE RHAM THEOREM FOR SYMPLECTIC QUOTIENTS
}

\author{
REYER SJAMAAR
}

\begin{abstract}
We introduce a de Rham model for stratified spaces arising from symplectic reduction. It turns out that the reduced symplectic form and its powers give rise to well-defined cohomology classes, even on a singular symplectic quotient.
\end{abstract}

\section{Introduction}

Let $G$ be a compact Lie group and let $M$ be a smooth $G$-manifold. Let $\Omega(M)$ be the de Rham complex of differential forms on $M$ and $\Omega_{\text {bas }}(M)$ the subcomplex of basic forms. It was proved by Koszul [1953] that the cohomology of $\Omega_{\mathrm{bas}}(M)$ is isomorphic to the cohomology with real coefficients of the orbit space $M / G$ (which is usually not a manifold, unless $G$ acts freely).

Now suppose that $M$ is equipped with a symplectic form $\omega$ and that the $G$-action is Hamiltonian with equivariant moment map $\Phi: M \rightarrow \mathfrak{g}^{*}$, where $\mathfrak{g}=\operatorname{Lie} G$. The appropriate quotient in this category is the Marsden-Meyer-Weinstein symplectic quotient $X=\Phi^{-1}(0) / G$. It is usually not a manifold either, unless $G$ acts freely on the fiber $\Phi^{-1}(0)$, but it always has a natural stratification into symplectic manifolds.

Much work has been done on the intersection cohomology of symplectic quotients; see, for example, [Kirwan 1985; Lerman and Tolman 2000]. The purpose of this note is rather more modest. We introduce a de Rham model for the ordinary cohomology of the symplectic quotient $X$, which is a straightforward adaptation of Koszul's complex of basic forms. It relies on a notion of a differential form on $X$ that extends the concept of a smooth function developed in [Arms et al. 1991]. Relevant examples are the reduced symplectic form and its powers, which define cohomology classes of even degree. These classes are nonzero if the quotient is compact. Thus the symplectic quotient, even when singular, carries a suitable analogue of a symplectic form and a Liouville volume form.

MSC2003: 53D20, 58A12.

Keywords: symplectic reduction, de Rham theory.

The author was partially supported by NSF Grant DMS-0071625. 


\section{Review}

Let $(M, \omega)$ be a connected symplectic manifold and let $G$ be a compact Lie group acting on $M$ in a Hamiltonian fashion with moment map $\Phi: M \rightarrow \mathfrak{g}^{*}$, where $\mathfrak{g}=$ Lie $G$. This means $d \Phi^{\xi}=i\left(\xi_{M}\right) \omega$, where $\xi_{M}$ denotes the vector field on $M$ induced by $\xi \in \mathfrak{g}$ and $\Phi^{\xi}=\langle\Phi, \xi\rangle$ denotes the component of the moment map along $\xi$. Also $\Phi$ is required to be equivariant with respect to the given action on $M$ and the coadjoint action on $\mathfrak{g}^{*}$. The symplectic quotient of $M$ by $G$ is the space $X=Z / G$, where $Z=\Phi^{-1}(0)$ is the zero fiber of the moment map. It was proved in [Marsden and Weinstein 1974] that if $G$ acts freely on $Z$, then $Z$ and $X$ are smooth manifolds and $X$ carries a natural symplectic form. If $G$ does not act freely on $Z$, often neither $Z$ nor $X$ are manifolds. In this case we proceed as in [Sjamaar and Lerman 1991], the relevant results of which we recall now. For any closed subgroup $H$ of $G$ let

$$
M_{(H)}=\left\{m \in M \mid G_{m} \text { is conjugate to } H\right\}
$$

be the stratum of orbit type $(H)$ in the $G$-manifold $M$. Here $G_{m}$ denotes the stabilizer of $m$ with respect to the $G$-action. Put

$$
Z_{(H)}=Z \cap M_{(H)} .
$$

Then $Z_{(H)}$ is a smooth $G$-stable submanifold of $M$. Let $\left\{Z_{a} \mid a \in A\right\}$ be the collection of connected components of all manifolds of the form $Z_{(H)}$, where $(H)$ ranges over all conjugacy classes of subgroups of $G$. The decomposition

$$
Z=\coprod_{a \in A} Z_{a}
$$

is a Whitney stratification of the fiber $Z$. In particular the index set $A$ has a partial order defined by $a \leq b$ if $Z_{a} \subseteq \bar{Z}_{b}$. There is a unique maximal element in $A$. The corresponding stratum, known as the principal or top stratum $Z_{\text {prin }}$, is open and dense in $Z$. Moreover the null foliation of the symplectic form $\omega$ restricted to any stratum $Z_{a}$ is exactly given by the $G$-orbits. Hence there exists a unique symplectic form $\omega_{a}$ on the quotient manifold $X_{a}=Z_{a} / G$ satisfying $\pi_{a}^{*} \omega_{a}=\iota_{a}^{*} \omega$, where $\iota_{a}: Z_{a} \hookrightarrow M$ is the inclusion map and $\pi_{a}: Z_{a} \rightarrow X_{a}$ the orbit map. The decomposition

$$
X=\coprod_{a \in A} X_{a}
$$

is a locally normally trivial stratification of the quotient $X$ into the symplectic manifolds $X_{a}$. The principal stratum $X_{\text {prin }}=Z_{\text {prin }} / G$ is open and dense in $X$. 


\section{Forms on a symplectic quotient}

We use the same notation as in the previous section. We denote the de Rham complex of a manifold $P$ by $\Omega(P)$. A differential form on the symplectic quotient $X$ is a differential form $\alpha$ on the top stratum $X_{\text {prin }}$ such that there exists a differential form $\tilde{\alpha}$ on $M$ satisfying $\pi_{\text {prin }}^{*} \alpha=\iota_{\text {prin }}^{*} \tilde{\alpha}$. We say that $\tilde{\alpha}$ induces $\alpha$. An easy averaging argument shows that we may assume $\tilde{\alpha}$ to be $G$-invariant on $M$. We denote the collection of differential forms on $X$ by $\Omega(X)$.

If $X=X_{\text {prin }}$, then $X$ and $Z$ are manifolds and the lift of any form on $X$ to $Z$ can be extended to $M$, so in this case our notion of a differential form on $X$ reduces to the standard notion. Observe that $\Omega(X)$ is a subcomplex of $\Omega\left(X_{\text {prin }}\right)$, and it is closed under the wedge product.

Example 3.1. The symplectic form $\omega_{\text {prin }}$ on $X_{\text {prin }}$ is induced by the symplectic form $\omega$ on $M$ and so defines a closed 2-form on $X$.

Clearly not every invariant form on $M$ induces a form on $X$. Indeed, if $\tilde{\alpha} \in$ $\Omega(M)^{G}$ induces $\alpha \in \Omega(X)$, then $\iota_{\text {prin }}^{*} \tilde{\alpha}=\pi_{\text {prin }}^{*} \alpha$ is a $G$-horizontal form on the $G$-manifold $Z_{\text {prin }}$, so it is annihilated by all inner products $i\left(\xi_{M}\right)$ for $\xi \in \mathfrak{g}$. Recall that a form $\beta$ on $M$ is basic with respect to the $G$-action if it is $G$-invariant and $G$-horizontal. Adapting this notion to our context, we say that $\beta$ is $\Phi$-basic if it is $G$-invariant and if $\iota_{\text {prin }}^{*} \beta \in \Omega\left(Z_{\text {prin }}\right)$ is horizontal. Let $\Omega_{\Phi}(M)$ denote the set of $\Phi$-basic forms. This is a subcomplex of $\Omega(M)$ and the kernel of the natural surjection $\Omega_{\Phi}(M) \rightarrow \Omega(X)$ is the ideal

$$
I_{\Phi}(M)=\left\{\beta \in \Omega(M)^{G} \mid \iota_{\text {prin }}^{*} \beta=0\right\} .
$$

Thus the de Rham complex of $X$ is isomorphic to

$$
\Omega(X) \cong \Omega_{\Phi}(M) / I_{\Phi}(M),
$$

a subquotient of the de Rham complex of $M$. In degree 0 we have the smooth functions on $X$ as defined in [Arms et al. 1991],

$$
C^{\infty}(X) \cong C^{\infty}(M)^{G} /\left\{f \in C^{\infty}(M)^{G} \mid f=0 \text { on } Z\right\} .
$$

If $O$ is a $G$-invariant open neighborhood of $Z$, then $O$ is a Hamiltonian $G$-manifold in its own right, so we can define $\Omega_{\Phi}(O)$ and $I_{\Phi}(O)$. Plainly (3.2) remains valid if we replace $M$ with $O$. Thus $\Omega(X)$ depends only on the $G$-germ of $M$ at $Z$.

It is true, though not completely obvious from the definition, that every form on $X$ restricts to a form on each stratum of $X$.

Lemma 3.3. (i) Let $\beta \in \Omega_{\Phi}(M)$. Then $\iota_{a}^{*} \beta$ is a horizontal form on $Z_{a}$ for all $a$.

(ii) Let $\beta \in I_{\Phi}(M)$. Then $\iota_{a}^{*} \beta=0$ for all $a$.

(iii) There is a well-defined restriction map $\Omega(X) \rightarrow \Omega\left(X_{a}\right)$ for each stratum $X_{a}$. 
Proof. Let $\beta \in \Omega_{\Phi}(M)$ and $z \in Z_{a}$. Choose a sequence $\left\{z_{n}\right\}$ in $Z_{\text {prin }}$ converging to $z$. By compactness of the Grassmannian we may assume that the sequence of tangent spaces $T_{z_{n}} Z_{\text {prin }}$ converges to a subspace $T$ of $T_{z} M$. By definition $i\left(\xi_{M}\right) \beta_{z_{n}}=0$ on $T_{z_{n}} Z_{\text {prin }}$ for all $\xi \in \mathfrak{g}$, so by continuity $i\left(\xi_{M}\right) \beta_{z}=0$ on $T$ for all $\xi$. By Whitney's Condition A we have $T_{z} Z_{a} \subseteq T$. Hence $i\left(\xi_{M}\right) \beta_{z}=0$ on $T_{z} Z_{a}$ for all $\xi$. This proves (i).

Similarly, if $\beta \in I_{\Phi}(M)$ then $\beta_{z_{n}}=0$ on $T_{z_{n}} Z_{\text {prin }}$, so by continuity $\beta_{z}=0$ on $T$ and hence $\beta_{z}=0$ on $T_{z} Z_{a}$, which proves (ii).

It follows from (i) that if $\beta \in \Omega_{\Phi}(M)$ then $\iota_{a}^{*} \beta$ descends to a form $\beta_{a}$ on $X_{a}$. The assignment $\beta \mapsto \beta_{a}$ defines a homomorphism $\Omega_{\Phi}(M) \rightarrow \Omega\left(X_{a}\right)$ for each $a$. It follows from (ii) that this map is 0 on the ideal $I_{\Phi}(M)$. Using the isomorphism (3.2) we obtain the desired restriction map $\Omega(X) \rightarrow \Omega\left(X_{a}\right)$.

\section{Symplectic induction}

A shortcoming of the de Rham complex $\Omega(X)$ is that it appears to depend on the way in which $X$ is written as a quotient. But in certain interesting situations this defect turns out to be illusory. For instance, let $H$ be a closed subgroup of $G$ and let $\left(N, \omega_{N}\right)$ be a Hamiltonian $H$-manifold with equivariant moment map $\Psi: N \rightarrow \mathfrak{h}^{*}$. Consider the Hamiltonian $G \times H$-space

$$
P=T^{*} G \times N
$$

where the action of $G$ on $P$ is given by left multiplication on $T^{*} G$ and the action of $H$ by right multiplication on $T^{*} G$ and the given action on $N$. Let $M$ be the symplectic quotient of $P$ with respect to the $H$-action. This is called the $G$ space induced by the $H$-space $N$. Since $H$ acts freely on $T^{*} G, M$ is a smooth manifold and from $P$ it inherits a symplectic form $\omega$ and a Hamiltonian $G$-action with moment map $\Phi$. Let $Y$ be the symplectic quotient of $N$ by the $H$-action and $X$ the symplectic quotient of $M$ by the $G$-action. The principle of reduction in stages implies that $X$ and $Y$ are isomorphic in the sense that there is a stratificationpreserving homeomorphism $Y \rightarrow X$ that restricts to a symplectomorphism on each stratum. We can represent the situation symbolically by a commutative diagram

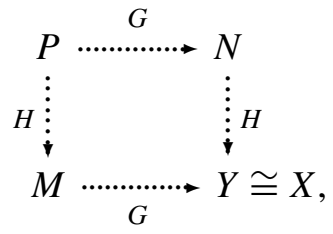

where the dotted arrows indicate symplectic reduction with respect to the relevant group. We assert that the de Rham complexes of $X$ and $Y$ are likewise isomorphic. 
To prove this we need to recall from [Sjamaar and Lerman 1991, §2] the definition of the isomorphism $Y \rightarrow X$. Choose an $H$-invariant subspace $\mathfrak{m}$ of $\mathfrak{g}$ complementary to the subalgebra $\mathfrak{h}$. Then we have $H$-invariant decompositions $\mathfrak{g}=\mathfrak{h} \oplus \mathfrak{m}$ and $\mathfrak{g}^{*}=\mathfrak{h}^{*} \oplus \mathfrak{m}^{*}$. Define a map

$$
G \times \mathfrak{m}^{*} \times N \rightarrow P \cong G \times \mathfrak{g}^{*} \times N
$$

by sending $(g, \alpha, p)$ to $(g, \alpha-\Psi(p), p)$. This is an $H$-equivariant diffeomorphism from $G \times \mathfrak{m}^{*} \times N$ onto the zero fiber of the $H$-moment map on $P$. Taking quotients by $H$ we obtain a $G$-equivariant diffeomorphism

$$
M \cong\left(G \times \mathfrak{m}^{*} \times N\right) / H
$$

from $M$ to the homogeneous vector bundle over $G / H$ with fiber $\mathfrak{m}^{*} \times N$. We identify $M$ with this bundle and write a typical point in it as $[g, \alpha, p]$, with $g \in G$, $\alpha \in \mathfrak{m}^{*}$ and $p \in N$. The $G$-action on $M$ is given by $k[g, \alpha, p]=[k g, \alpha, p]$ for $k \in G$ and the moment map by

$$
\Phi([g, \alpha, p])=\operatorname{Ad}^{*}(g)(\alpha+\Psi(p)) .
$$

Let $f: N \rightarrow M$ be the embedding defined by $f(p)=[1,0, p]$. Then $f$ is $H$ equivariant and (4.1) shows that $\Phi \circ f=\operatorname{pr}^{*} \circ \Psi$, where $\mathrm{pr}^{*}: \mathfrak{h}^{*} \rightarrow \mathfrak{g}^{*}$ is the transpose of the projection map $\mathfrak{g} \rightarrow \mathfrak{h}$. Hence $f$ maps the zero fiber $Z_{N}=\Psi^{-1}(0)$ into $Z$ and descends to a map $Y \rightarrow X$, which is the required isomorphism. In particular $f$ maps the principal stratum $\left(Z_{N}\right)_{\text {prin }}$ into the principal stratum of $Z$. In fact $Z$ and $Z_{\text {prin }}$ are homogeneous bundles over $G / H$,

$$
Z=\left(G \times Z_{N}\right) / H \quad \text { and } \quad Z_{\text {prin }}=\left(G \times\left(Z_{N}\right)_{\text {prin }}\right) / H .
$$

This implies that the restriction map $f^{*}: \Omega(M) \rightarrow \Omega(N)$ sends $\Omega_{\Phi}(M)$ to $\Omega_{\Psi}(N)$ and $I_{\Phi}(M)$ to $I_{\Psi}(N)$. Therefore, because of the isomorphism (3.2), it descends to a map $r: \Omega(X) \rightarrow \Omega(Y)$.

Proposition 4.2. The map $r: \Omega(X) \rightarrow \Omega(Y)$ is an isomorphism.

Proof. This relies on material developed in the Appendix. Let $\iota_{\text {prin }}: Z_{\text {prin }} \rightarrow M$ be the inclusion map. This is a bundle map of fiber bundles over the base $G / H$. Its restriction to a fiber is the inclusion map $\left(\iota_{N}\right)_{\text {prin }}:\left(Z_{N}\right)_{\text {prin }} \rightarrow N$. Let

$$
\begin{aligned}
& e_{M}: \Omega(N)^{H} \rightarrow \Omega(M)^{G}, \\
& e_{Z}: \Omega\left(\left(Z_{N}\right)_{\text {prin }}\right)^{H} \rightarrow \Omega\left(Z_{\text {prin }}\right)^{G}
\end{aligned}
$$

be the extension homomorphisms for the homogeneous bundles $M$ and $Z_{\text {prin }}$ as defined in the Appendix. Then

$$
e_{Z} \circ\left(\iota_{N}\right)_{\text {prin }}^{*}=\iota_{\text {prin }}^{*} \circ e_{M}
$$


by Lemma A.2.

Now we show that $r$ is surjective. In fact we must show that $f^{*} \Omega_{\Phi}(M)=$ $\Omega_{\Psi}(N)$. Let $\gamma \in \Omega_{\Psi}(N)$. Then by definition $\left(\iota_{N}\right)_{\text {prin }}^{*} \gamma$ is $H$-basic, so $e_{Z}\left(\left(\iota_{N}\right)_{\text {prin }}^{*} \gamma\right)$ is $G$-basic by Lemma A.1(ii). From (4.3) we get that $\iota_{\text {prin }}^{*} e_{M}(\gamma)$ is $G$-basic, i.e. $e_{M}(\gamma) \in \Omega_{\Phi}(M)$. Using Lemma A.1(i) we find that $\gamma=f^{*} \beta$ with $\beta=e_{M}(\gamma) \in$ $\Omega_{\Phi}(M)$. Hence $f^{*} \Omega_{\Phi}(M)=\Omega_{\Psi}(N)$.

Next we prove that $r$ is injective. Suppose that $\beta \in \Omega_{\Phi}(M)$ satisfies $f^{*} \beta \in$ $I_{\Psi}(N)$. We need to show that $\beta \in I_{\Phi}(M)$. The assumptions on $\beta$ mean that $\iota_{\text {prin }}^{*} \beta$ is $G$-basic and that $\left(\iota_{N}\right)_{\text {prin }}^{*} f^{*} \beta=0$. Using Lemma A.1(iii) we get

$$
\iota_{\mathrm{prin}}^{*} \beta=e_{Z}\left(f^{*} \iota_{\mathrm{prin}}^{*} \beta\right)=e_{Z}\left(\left(\iota_{N}\right)_{\mathrm{prin}}^{*} f^{*} \beta\right)=e_{Z}(0)=0,
$$

that is, $\beta \in I_{\Phi}(M)$.

\section{The de Rham sheaf}

To prove a de Rham theorem we need to sheafify the de Rham complex. Let $U$ be an open subset of the symplectic quotient $X$. The stratification of $X$ induces one on $U$, so we can talk about the principal stratum of $U$ etc. A differential form on $U$ is a differential form $\alpha$ on $U_{\text {prin }}$ such that for all $x \in U$ there exist $\alpha^{\prime} \in \Omega(X)$ and an open neighborhood $U^{\prime}$ of $x$ in $U$ such that $\alpha=\alpha^{\prime}$ on $U_{\text {prin }}^{\prime}$. The set of differential forms on $U$ is denoted by $\Omega(U)$. It is easy to check that the presheaf of differential graded algebras $\Omega: U \mapsto \Omega(U)$ is a sheaf. Its space of global sections is the previously defined de Rham complex $\Omega(X)$.

Lemma 5.1. $\Omega$ is an acyclic sheaf, i.e. $H^{i}\left(X, \Omega^{j}\right)=0$ for all $i \geq 1$ and $j \geq 0$.

Proof. The space $X$ possesses smooth partitions of unity subordinate to arbitrary open covers $U$. Indeed, for each $U \in U$ choose a $G$-invariant open $\tilde{U}$ in $M$ such that $U=(\tilde{U} \cap Z) / G$ and let $O$ be the union of the $\tilde{U}$ 's. Choose a smooth $G$-invariant partition of unity on the $G$-manifold $O$ subordinate to the cover defined by the $\tilde{U}$ 's; this induces a smooth partition of unity on $X$ subordinate to $U$. Thus the sheaf of smooth functions $\Omega^{0}$ is fine in the sense of [Godement 1973, §3.7]. A standard result in sheaf theory (see [Godement 1973, Théorème 4.4.3], for example) now implies that $\Omega^{0}$ is acyclic. Since $\Omega$ is a module over $\Omega^{0}$, it is fine, and therefore acyclic, as well.

There is an alternative characterization of forms on open subsets of $X$. The proof is an easy exercise involving partitions of unity.

Lemma 5.2. Let $U$ be an open subset of $X$ and let $\alpha \in \Omega\left(U_{\text {prin }}\right)$. Then $\alpha \in \Omega(U)$ if and only if there exist a $G$-invariant open subset $\tilde{U}$ of $M$ and a form $\tilde{\alpha} \in \Omega(\tilde{U})$ such that $U=(\tilde{U} \cap Z) / G$ and $\iota_{\text {prin }}^{*} \tilde{\alpha}=\pi_{\text {prin }}^{*} \alpha$. 
Now let $\underline{\mathbb{R}}$ be the sheaf of locally constant real-valued functions on $X$ and consider the sequence

$$
0 \rightarrow \underline{\mathbb{R}} \stackrel{i}{\rightarrow} \Omega^{0} \stackrel{d}{\rightarrow} \Omega^{1} \stackrel{d}{\rightarrow} \cdots,
$$

where $i: \underline{\mathbb{R}} \rightarrow \Omega^{0}$ is the natural inclusion. The following assertion is proved in the next section.

Lemma 5.4. The sequence (5.3) is exact.

Thus the de Rham complex is an acyclic resolution of the constant sheaf, which by standard sheaf theory (see [Godement 1973, Théorèmes 4.7.1, 6.2.1], for example) implies the following de Rham theorem.

Theorem 5.5. The de Rham cohomology ring $H(\Omega(X))$ is naturally isomorphic to the $(\check{C}$ ech or singular) cohomology ring of $X$ with real coefficients $H(X, \mathbb{R})$.

\section{The Poincaré lemma}

In this section we prove the following (marginally stronger) version of Lemma 5.4: every $x \in X$ has a basis of open neighborhoods $U$ such that the sequence

$$
0 \rightarrow \mathbb{R} \stackrel{i}{\rightarrow} \Omega^{0}(U) \stackrel{d}{\rightarrow} \Omega^{1}(U) \stackrel{d}{\rightarrow} \cdots
$$

is exact. The proof is a variation on a familiar homotopy argument in de Rham theory, which requires a brief look into the functorial properties of $\Omega(X)$.

Let $\left(M^{\prime}, \omega^{\prime}, \Phi^{\prime}\right)$ be a second Hamiltonian $G$-manifold with zero fiber $Z^{\prime}=$ $\left(\Phi^{\prime}\right)^{-1}(0)$ and symplectic quotient $X^{\prime}=Z^{\prime} / G$. Then we have stratifications $Z^{\prime}=$ $\bigsqcup_{a \in A^{\prime}} Z_{a}^{\prime}$ and $X^{\prime}=\bigsqcup_{a \in A^{\prime}} X_{a}^{\prime}$ analogous to those for $Z$ and $X$. Call a map $f: M \rightarrow$ $M^{\prime}$ allowable if

(i) $f$ is smooth and $G$-equivariant;

(ii) $f(Z) \subseteq Z^{\prime}$;

(iii) $d f\left(T_{z} Z_{\text {prin }}\right) \subseteq T_{f(z)} Z_{a(z)}^{\prime}$ for all $z \in Z_{\text {prin }}$, where $Z_{a(z)}^{\prime} \subseteq Z^{\prime}$ is the stratum of $f(z)$.

For instance, if $f$ is smooth and equivariant and maps $Z_{\text {prin }}$ into a single stratum of $Z^{\prime}$, then $f$ is allowable.

Example 6.2. Let $(V, \omega)$ be a symplectic vector space on which $G$ acts linearly and symplectically. A moment map is given by $\Phi_{V}^{\xi}(v)=\frac{1}{2} \omega(\xi v, v)$, where $\xi \in \mathfrak{g}$ acts on $V$ via the infinitesimal representation $\mathfrak{g} \rightarrow \mathfrak{s p}(V)$. Let $t \in \mathbb{R}$ and let $f: V \rightarrow V$ be the dilation $f(v)=t v$. Clearly $f$ preserves $Z$. Furthermore, if $t \neq 0$, then $f(v)$ has the same stabilizer as $v$, so $f$ maps $Z_{\text {prin }}$ to itself. If $t=0$, then $f$ maps $Z_{\text {prin }}$ to 0 . In either case $f$ maps $Z_{\text {prin }}$ into a single stratum of $Z$ and it is obviously 
smooth and equivariant, so it is allowable. Similarly, if $|t| \leq 1$ and $B$ is a $G$ invariant open ball about the origin, the restriction of $f$ is an allowable map from $B$ to itself.

The following result is easy to deduce from Lemma 3.3.

Lemma 6.3. Let $f: M \rightarrow M^{\prime}$ allowable. Then the pullback homomorphism $f^{*}: \Omega\left(M^{\prime}\right) \rightarrow \Omega(M)$ sends $\Omega_{\Phi^{\prime}}\left(M^{\prime}\right)$ to $\Omega_{\Phi}(M)$ and $I_{\Phi^{\prime}}\left(M^{\prime}\right)$ to $I_{\Phi}(M)$, and therefore induces a homomorphism $f^{*}: \Omega\left(X^{\prime}\right) \rightarrow \Omega(X)$.

Homotopies induce chain homotopies on the de Rham complex in a standard way. Let $F: M \times[0,1] \rightarrow M^{\prime}$ be a smooth homotopy and put $F_{t}=\left.F\right|_{M \times\{t\}}$. Let $t$ be the coordinate on $[0,1]$ and for $\gamma \in \Omega\left(M^{\prime}\right)$ put $\kappa_{F} \gamma=\int_{0}^{1} i(\partial / \partial t) F^{*} \gamma d t$. Then $\kappa_{F}$ lowers the degree by 1 and

$$
F_{1}^{*}-F_{0}^{*}=\kappa_{F} d+d \kappa_{F} .
$$

Assume that $F$ is equivariant with respect to the given $G$-actions on $M$ and $M^{\prime}$ and the trivial action on $[0,1]$. It is straightforward to check that

$$
\begin{aligned}
\kappa_{F} \circ g^{*} & =g^{*} \circ \kappa_{F} & & \text { for all } g \in G, \\
\kappa_{F} \circ i\left(\xi_{M^{\prime}}\right) & =-i\left(\xi_{M}\right) \circ \kappa_{F} & & \text { for all } \xi \in \mathfrak{g} .
\end{aligned}
$$

Call the homotopy $F$ allowable if

(i) $F$ is smooth and $G$-equivariant;

(ii) $F_{t}: M \rightarrow M^{\prime}$ is allowable for almost all $t \in[0,1]$;

(iii) $d F_{(z, t)}(\partial / \partial t) \in T_{F(z, t)} Z_{a(z, t)}^{\prime}$ for almost all $t \in[0,1]$ and for all $z \in Z_{\text {prin }}$, where $Z_{a(z, t)}^{\prime} \subseteq Z^{\prime}$ is the stratum of $F(z, t)$.

For instance, if $F$ is smooth and equivariant and if there exists a single stratum $Z_{a}^{\prime}$ of $Z^{\prime}$ such that $F_{t}\left(Z_{\text {prin }}\right) \subseteq Z_{a}^{\prime}$ for almost all $t$, then $F$ is allowable.

Example 6.6. Let $(V, \omega)$ be a symplectic representation space for $G$ as in Example 6.2. The radial contraction $F: V \times[0,1] \rightarrow V$ given by $F(v, t)=t v$ is smooth and equivariant and satisfies $F_{t}\left(Z_{\text {prin }}\right) \subseteq Z_{\text {prin }}$ for $t \neq 0$. Hence it is allowable. Likewise, $F$ defines an allowable homotopy $B \times[0,1] \rightarrow B$ for any $G$-invariant open ball $B$ about the origin.

Lemma 6.7. Let $F: M \times[0,1] \rightarrow M^{\prime}$ be an allowable homotopy. Then the homotopy operator $\kappa_{F}: \Omega\left(M^{\prime}\right) \rightarrow \Omega(M)$ sends $\Omega_{\Phi^{\prime}}\left(M^{\prime}\right)$ to $\Omega_{\Phi}(M)$ and $I_{\Phi^{\prime}}\left(M^{\prime}\right)$ to $I_{\Phi}(M)$, and therefore induces a homotopy $\kappa_{F}: \Omega\left(X^{\prime}\right) \rightarrow \Omega(X)$.

Proof. Let $\gamma \in \Omega_{\Phi^{\prime}}^{k}\left(M^{\prime}\right)$. Then $\gamma$ is invariant, so $\kappa_{F} \gamma$ is invariant by (6.4). Let $z \in Z_{\text {prin. }}$. Using (6.5) we find that for any multivector $v \in \Lambda^{k-1}\left(T_{z} Z_{\text {prin }}\right)$

$$
i\left(\xi_{M}\right)\left(\kappa_{F} \gamma\right)_{z}(v)=\int_{0}^{1} \phi(t) d t
$$


where $\phi(t)=-\gamma_{F(z, t)}\left(\xi_{M^{\prime}}, F_{*} \partial / \partial t,\left(F_{t}\right)_{*} v\right)$. Let $Z_{a(z, t)}^{\prime}$ be the stratum of $Z^{\prime}$ containing $F(z, t)$. Since $F$ is allowable,

$$
F_{*} \partial / \partial t \in T_{F(z, t)} Z_{a(z, t)}^{\prime} \quad \text { and } \quad\left(F_{t}\right)_{*} v \in \Lambda^{k-1}\left(T_{F(z, t)} Z_{a(z, t)}^{\prime}\right)
$$

for most $t$. Moreover, by Lemma 3.3(i) the restriction of $\gamma$ to $Z_{a(z, t)}^{\prime}$ is horizontal. Hence $\phi(t)=0$ for almost all $t$. From (6.8) we get $i\left(\xi_{M}\right)\left(\kappa_{F} \gamma\right)_{z}(v)=0$; in other words $\kappa_{F} \gamma \in \Omega_{\Phi}^{k-1}(M)$. The inclusion $\kappa_{F} I_{\Phi^{\prime}}\left(M^{\prime}\right) \subseteq I_{\Phi}(M)$ is proved in a similar way, and the last assertion now follows from the isomorphism (3.2).

Example 6.9. Applying Lemma 6.7 to the radial contraction of Example 6.6 we find that the de Rham complex of the symplectic quotient of a vector space $V$ is homotopically trivial. More generally, if $Y=(B \cap Z) / G$ is the symplectic quotient of any $G$-invariant open ball $B$ about the origin, then the de Rham complex of $Y$ is homotopically trivial.

Example 6.10. Let $H$ be a closed subgroup of $G$ and let $V$ be a symplectic $H$ module. Let $B$ be an $H$-invariant open ball about the origin and let $O$ be the Hamiltonian $G$-manifold induced by $B$. Let $Y$ be the symplectic quotient of $B$ by the $H$-action and $U$ the symplectic quotient of $O$ by the $G$-action. Then $\Omega(U) \cong$ $\Omega(Y)$ by Proposition 4.2, so $\Omega(U)$ is homotopically trivial by Example 6.9.

This example generalizes to arbitrary Hamiltonian $G$-manifolds by means of a slice argument. Let $z \in Z$ and let $H=G_{z}$ be the stabilizer of $z$. Consider the symplectic $H$-module $V=\left(T_{z} G z\right)^{\omega} / T_{z} G z$ known as the symplectic slice at $z$. Choose an $H$-invariant open ball $B$ in $V$ and let $O$ be the $G$-space induced by $B$. The symplectic slice theorem due to Marle and to Guillemin and Sternberg (see [Sjamaar and Lerman 1991, §2], for instance) says that, for sufficiently small $B$, $z$ has a $G$-invariant open neighborhood that is isomorphic to $O$ as a Hamiltonian $G$-manifold. Hence the point $x \in X$ determined by $z$ has an open neighborhood $U$ for which $\Omega(U)$ is homotopically trivial. By letting $B$ shrink to a point we obtain a collection of such neighborhoods, which is a basis of the topology at $x$. This finishes the proof of (6.1).

\section{Integration and the symplectic class}

In this section we show that top-degree forms on a compact symplectic quotient are always integrable and establish a version of Stokes' theorem. We conclude that the cohomology class of the symplectic form and its powers are nonzero.

For technical reasons we do not assume at the outset that $X$ is compact. We start by introducing a metric on $X_{\text {prin }}$ and demonstrating that $X$ has "locally finite" volume. Choose a $G$-invariant compatible almost complex structure $J$ on the Hamiltonian $G$-manifold $M$. The volume element determined by the Riemannian metric $\sigma=\omega\left(\cdot, J \cdot\right.$ ) is identical to the Liouville volume form $\omega^{d} / d$ ! (where 
$2 d=\operatorname{dim} M)$. The almost complex structure and Riemannian metric descend in a natural way to each stratum of $X$. Let $2 n=\operatorname{dim} X$ and write $\mu=\omega_{\text {prin }}^{n} / n$ ! for the volume element of the principal stratum $X_{\text {prin }}$.

Lemma 7.1. Every $x \in X$ has an open neighborhood $U$ such that vol $U_{\text {prin }}$ is finite. Hence $X_{\text {prin }}$ has finite volume if $X$ is compact.

Proof. Choose $z \in Z$ mapping to $x$ and let $H=G_{z}$. By the symplectic slice theorem we may take $U$ to be the symplectic quotient of an $H$-invariant neighborhood $B$ of the origin in the symplectic slice $V$ at $z$. The almost complex structure on $M$ induces one on $V$, turning $V$ into a unitary $H$-module. The metric on $U_{\text {prin }}$ induced by the flat metric $\sigma_{V}$ on $V$ is quasi-isometric to the metric induced by $\sigma$. Therefore it is enough to show that $U$ has finite volume with respect to the former. Let $W$ be the orthogonal complement in $V$ of the subspace of invariants $V^{H}$. The quadratic moment map $\Phi_{V}$ is constant along $V^{H}$, so $Z_{V}=V^{H} \times Z_{W}$, where $Z_{V}=\Phi_{V}^{-1}(0)$ and $Z_{W}=\Phi_{V}^{-1}(0) \cap W$. Let $B=B_{1} \times B_{2}$, where $B_{1}$ is an open ball about the origin in $V^{H}$ and $B_{2}$ an $H$-invariant open ball about the origin in $W$. Then $B$ has a product metric and so do $\left(Z_{V}\right)_{\text {prin }}=V^{H} \times\left(Z_{W}\right)_{\text {prin }}$ and the quotient

$$
U_{\text {prin }}=B_{1} \times\left(B_{2} \cap\left(Z_{W}\right)_{\text {prin }}\right) / H .
$$

Recall that the metric cone over a Riemannian manifold $\left(Y, \sigma_{Y}\right)$ is the product $Y \times(0,1)$ with metric $t^{2} \sigma_{Y}+d t \otimes d t$, where $t$ is the coordinate on $(0,1)$. The metric cone over $Y$ has finite volume if $Y$ does. For instance, the ball $B_{2}$ in $W$ is the metric cone over the sphere $S=\partial B_{2}$. Similarly, with respect to the metric induced by $\sigma_{W}, B_{2} \cap\left(Z_{W}\right)_{\text {prin }}$ is a metric cone over $S \cap\left(Z_{W}\right)_{\text {prin. }}$. Upon taking quotients we see that $\left(B_{2} \cap\left(Z_{W}\right)_{\text {prin }}\right) / H$ is a metric cone over $\left(S \cap\left(Z_{W}\right)_{\text {prin }}\right) / H$. The link $S \cap Z_{W}$ is the zero fiber of the moment map $v \mapsto\left(\Phi_{W}(v), \frac{1}{2}\left(1-|v|^{2}\right)\right)$ for the $H \times \mathbf{U}(1)$-action on $W$, where $\mathbf{U}(1)$ acts by complex scalar multiplication. By induction on the depth of the stratification, the principal stratum of the symplectic quotient $\left(S \cap\left(Z_{W}\right)\right) / H$ has finite volume. Hence $\left(B_{2} \cap\left(Z_{W}\right)_{\text {prin }}\right) / H$ has finite volume and therefore, because of the product decomposition (7.2), so does $U_{\text {prin }}$.

The Riemannian metric on $M$ determines metrics on $\Lambda^{k}(T M)$ for all $k$. Let $|\beta| \in C^{0}(M)$ denote the pointwise norm of a form $\beta$ on $M$. Similarly, for $\alpha \in \Omega(X)$ let $|\alpha| \in C^{0}\left(X_{\text {prin }}\right)$ denote the pointwise norm over the principal stratum. If $\alpha$ is induced by $\tilde{\alpha} \in \Omega_{\Phi}(M)$, then $|\tilde{\alpha}|$ is a $G$-invariant continuous function on $M$ and

$$
\pi_{\text {prin }}^{*}|\alpha| \leq \iota_{\text {prin }}^{*}|\tilde{\alpha}| .
$$

The support of a form $\alpha \in \Omega(X)$ is its support as a section of the sheaf $\Omega$. This is the same as the closure in $X$ of the support of $\alpha$ considered as a form on $X_{\text {prin }}$. The estimate (7.3) implies that for $\alpha \in \Omega(X)$ with compact support the pointwise 
norm $|\alpha|$ is a bounded function on $X_{\text {prin }}$ and therefore by Lemma 7.1 the global norm $\int_{X_{\text {prin }}}|\alpha| \mu$ is finite. In particular, for $\alpha$ of top degree $2 n$ the integral $\int_{X_{\text {prin }}} \alpha$ is absolutely convergent.

We can now prove Stokes' theorem. The proof is based on the fact that the singular strata of $X$ have codimension $\geq 2$, which makes the boundary terms in the integral vanish.

Proposition 7.4. $\int_{X_{\text {prin }}} d \gamma=0$ if $\gamma \in \Omega^{2 n-1}(X)$ has compact support.

Proof. We use the notation of the proof of Lemma 7.1. By using partitions of unity we can reduce the general case to the case where $\gamma$ has compact support in an open subset $U$ of the form $B_{1} \times\left(B_{2} \cap Z_{W}\right) / H$. Let $2 m=\operatorname{dim} Z_{W} / H$. If $m=0$ then $U$ is nonsingular and the result follows from the usual version of Stokes' theorem, so we may assume $m \geq 1$. Let $\chi:[0, \infty) \rightarrow[0,1]$ be a smooth function satisfying $\chi(t)=0$ for $t$ near 0 and $\chi(t)=1$ for $t \geq 1$. Define a sequence of $H$-invariant functions $\tilde{\chi}_{k}: V \rightarrow[0,1]$ for $k \geq 1$ by $\tilde{\chi}_{k}(v)=\chi\left(k\left|\operatorname{pr}_{W} v\right|\right)$, where $\operatorname{pr}_{W}: V \rightarrow W$ is the orthogonal projection. These functions descend to smooth functions $\chi_{k}: U \rightarrow[0,1]$. The functions $1-\chi_{k}$ are bump functions supported near the singularities of $U$. In fact the sets $S_{k}=\operatorname{supp}\left(1-\chi_{k}\right)$ form a decreasing sequence satisfying

$$
\bigcap_{k} S_{k}=B_{1} \times\{0 \bmod H\},
$$

the most singular stratum of $U$. Therefore $\bigcap_{k}\left(S_{k}\right)_{\text {prin }}$ is empty and

$$
\left|\int_{X_{\text {prin }}} d \gamma-\int_{X_{\text {prin }}} \chi_{k} d \gamma\right|=\left|\int_{\left(S_{k}\right)_{\text {prin }}}\left(1-\chi_{k}\right) d \gamma\right| \leq C \operatorname{vol}\left(S_{k}\right)_{\text {prin }} \rightarrow 0
$$

as $k \rightarrow \infty$. (Here $C$ is an upper bound for $\left|\left(1-\chi_{k}\right) d \gamma\right|$.) This shows that

$$
\int_{X_{\text {prin }}} d \gamma=\lim _{k \rightarrow \infty} \int_{X_{\text {prin }}} \chi_{k} d \gamma
$$

To see that this limit is 0 we use

$$
\int_{X_{\text {prin }}} \chi_{k} d \gamma=\int_{X_{\text {prin }}} d\left(\chi_{k} \gamma\right)-\int_{X_{\text {prin }}} d \chi_{k} \wedge \gamma .
$$

Since $d\left(\chi_{k} \gamma\right)$ is supported away from the most singular stratum (7.5), we can assume by induction on the depth of the stratification that $\int_{X_{\text {prin }}} d\left(\chi_{k} \gamma\right)=0$. Moreover,

$$
\left|\int_{X_{\text {prin }}} d \chi_{k} \wedge \gamma\right| \leq \int_{X_{\text {prin }}}\left|d \chi_{k}\right||\gamma| \mu \leq C \int_{\left(S_{k}\right)_{\text {prin }}}\left|d \chi_{k}\right| \mu,
$$

where $C$ is an upper bound for $|\gamma|$. Let $\tilde{\rho}_{k}: W \rightarrow W$ be the dilation $v \mapsto k v$ and $\rho_{k}$ the induced map on $Z_{V} / H$. Then $\chi_{k}=\chi_{1} \circ \rho_{k}$ and $S_{k}=\rho_{k}^{-1}\left(S_{1}\right)$. It follows 
that $d \chi_{k}(x)=k d \chi_{1}\left(\rho_{k}(x)\right)$. By (7.2), $U_{\text {prin }}$ is the product of a ball and a metric cone, so $\operatorname{vol}\left(S_{k}\right)_{\text {prin }}=k^{-2 m} \operatorname{vol}\left(S_{1}\right)_{\text {prin }}$, where $2 m=\operatorname{dim} Z_{W} / H \geq 2$. Hence

$$
\left|\int_{X_{\text {prin }}} d \chi_{k} \wedge \gamma\right| \leq C k^{1-2 m} \int_{\left(S_{1}\right)_{\text {prin }}}\left|d \chi_{1}\right| \mu \rightarrow 0
$$

as $k \rightarrow \infty$. Therefore $\lim _{k \rightarrow \infty} \int_{X_{\text {prin }}} \chi_{k} d \gamma=0$.

Stokes' theorem implies that the volume form of a compact quotient is not exact. Corollary 7.6. Suppose that $X$ is compact. Then the class of $\omega_{\text {prin }}^{k}$ in $H^{2 k}(\Omega(X))$ is nonzero for $0 \leq k \leq n$, where $2 n=\operatorname{dim} X$.

\section{Generalizations}

The results above can be generalized in two obvious ways. First we consider symplectic quotients at nonzero levels. Let $\mathcal{O}$ be a coadjoint orbit in $\mathfrak{g}^{*}$. The symplectic quotient at $\mathcal{O}$ is $X_{\overparen{O}}=Z_{\mathscr{O}} / G$, where $Z_{\overparen{O}}$ is the fiber $\Phi^{-1}(\mathcal{O})$. The spaces $Z_{\overparen{O}}$ and $X_{\overparen{O}}$ stratify in exactly the same way as when $O=\{0\}$ and the strata of $X_{\mathscr{O}}$ again carry natural symplectic forms. Differential forms on $X_{\mathscr{O}}$ can now be defined as before. There is a symplectic slice theorem for orbits in $Z_{\mathscr{O}}$, so all our results generalize to this situation with virtually unchanged proofs.

Next we consider actions of a noncompact group $G$. The symplectic slice theorem remains valid, provided that $G$ acts properly on $M$. For locally closed coadjoint orbits 0 stratifications of $Z_{\mathscr{O}}$ and $X_{\mathscr{O}}$ were obtained in [Bates and Lerman 1997]. However, our definition of forms on $X$ is valid as it stands only when $O$ is closed, because forms on a nonclosed subset may not extend to the ambient manifold. If

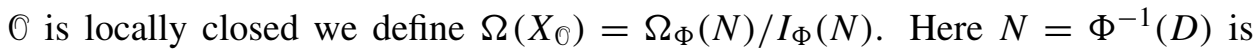
the preimage of any $G$-invariant open neighborhood $D$ of $\mathcal{O}$ in $\mathfrak{g}^{*}$ such that $\mathcal{O}$ is closed in $D, \Omega_{\Phi}(N)$ is the set of $G$-invariant forms on $N$ that restrict to basic

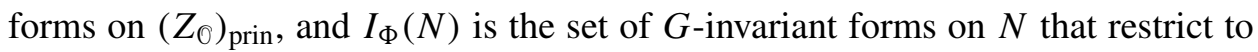
0 on $\left(Z_{\overparen{O}}\right)_{\text {prin }}$. With this minor modification our results carry over to symplectic quotients by proper actions at locally closed coadjoint orbits. (For general orbits one might try to apply the methods developed in [Cushman and Śniatycki 2001], but we have not attempted this.)

\section{Appendix: Forms on homogeneous bundles}

Let $G$ be a compact Lie group and $H$ a closed subgroup. For any $H$-manifold $F$ we can form the homogeneous fiber bundle with fiber $F$ over $G / H$,

$$
E=(G \times F) / H .
$$

The map $f: F \rightarrow E$ defined by $f(p)=[1, p]$ identifies $F$ with the fiber over the coset $0 \bmod H$. (Here $[g, p]$ denotes the coset $(g, p) \bmod H$ of $(g, p) \in G \times F$.) 
Restriction to the fiber is a homomorphism

$$
f^{*}: \Omega(E)^{G} \rightarrow \Omega(F)^{H} .
$$

It is not hard to see that $G$-basic forms on $E$ restrict to $H$-basic forms on $F$ and that $f^{*}: \Omega_{\mathrm{bas}}(E) \rightarrow \Omega_{\mathrm{bas}}(F)$ is an isomorphism. We require a slight generalization of this elementary fact.

Choose an $H$-equivariant projection $\mathfrak{g} \rightarrow \mathfrak{h}$; this determines a $G$-invariant connection 1-form $\theta \in \Omega^{1}(G, \mathfrak{h})^{G \times H}$ on the principal $H$-bundle $G \rightarrow G / H$. Let $V E$ be the vertical tangent bundle of $E$ and let $\theta_{E} \in \Omega^{1}(E, V E)^{G}$ be the $G$-invariant connection 1-form on $E$ associated to $\theta$. Let $\gamma \in \Omega(F)^{H}$ be any invariant form on the fiber. Define a form $e(\gamma) \in \Omega(E)$ by putting

$$
e(\gamma)_{[g, p]}(v)=\gamma_{p}\left(\left(g^{-1}\right)_{*} \theta_{E}(v)\right)
$$

for $[g, p] \in E$ and $v \in \Lambda\left(T_{[g, p]} E\right)$. (For simplicity we write $\theta_{E}$ for the extension of the connection $\theta_{E}: T E \rightarrow V E$ to a multiplicative map $\Lambda(T E) \rightarrow \Lambda(V E)$.) The $H$-invariance of $\gamma$ implies that $e(\gamma)_{[g, p]}(v)$ does not depend on the choice of the representative $(g, p)$ of the coset $[g, p]$. The $G$-invariance of $\theta_{E}$ implies that $e(\gamma)$ is $G$-invariant. Thus we have defined a map

$$
e: \Omega(F)^{H} \rightarrow \Omega(E)^{G},
$$

which we call the extension homomorphism determined by $\theta$. (An alternative definition runs as follows. Let $\mathscr{V}=\operatorname{pr}^{*} T F$, where pr: $G \times F \rightarrow F$ is the Cartesian projection. The vertical bundle of $E$ is then the quotient $V E \cong \mathscr{V} / H$. A form $\gamma \in \Omega(F)^{H}$ is a section of $\Lambda(T F)$ and as such extends uniquely to a section $\tilde{\gamma}$ of $\mathscr{V}$ that is constant along $G$. Then $\tilde{\gamma}$ is $G \times H$-invariant and so descends to a $G$-invariant section $\bar{\gamma}$ of $V E$. Thus $e(\gamma)=\theta_{E}^{*} \bar{\gamma}$ is a $G$-invariant section of $\Lambda(T E)$. This argument also shows that $e(\gamma)$ is smooth.) The following result is immediate from the definition.

Lemma A.1. (i) $f^{*} e(\gamma)=\gamma$ for $\gamma \in \Omega(F)^{H}$;

(ii) e maps $\Omega(F)_{\text {bas }}$ to $\Omega(E)_{\text {bas }}$;

(iii) $e\left(f^{*} \beta\right)=\beta$ for $\beta \in \Omega(E)_{\text {bas }}$.

It follows from (i) that $f^{*}: \Omega(E)^{G} \rightarrow \Omega(F)^{H}$ is surjective and from (ii)-(iii) that $f^{*}: \Omega_{\text {bas }}(E) \rightarrow \Omega_{\text {bas }}(F)$ is an isomorphism, as noted above. Now let $F^{\prime}$ be a second $H$-manifold and let $j: F \rightarrow F^{\prime}$ be an $H$-equivariant map. Then $j$ extends naturally to an $G$-equivariant bundle map $\bar{j}: E \rightarrow E^{\prime}=\left(G \times F^{\prime}\right) / H$. Moreover $\theta_{E}$ is the pullback of the associated connection $\theta_{E^{\prime}}$ on $E^{\prime}$. This implies that the extension homomorphism is functorial in the following sense.

Lemma A.2. $e \circ j^{*}=\bar{j}^{*} \circ e^{\prime}$, where $e^{\prime}: \Omega\left(F^{\prime}\right)^{H} \rightarrow \Omega\left(E^{\prime}\right)^{G}$ is the extension homomorphism for $E^{\prime}$. 


\section{References}

[Arms et al. 1991] J. M. Arms, R. H. Cushman, and M. J. Gotay, "A universal reduction procedure for Hamiltonian group actions", pp. 33-51 in The geometry of Hamiltonian systems (Berkeley, 1989), edited by T. Ratiu, Math. Sci. Res. Inst. Publ. 22, Springer, New York, 1991. MR 92h:58059 Zbl 0742.58016

[Bates and Lerman 1997] L. Bates and E. Lerman, "Proper group actions and symplectic stratified spaces”, Pacific J. Math. 181:2 (1997), 201-229. MR 98i:58085 Zbl 0902.58008

[Cushman and Śniatycki 2001] R. Cushman and J. Śniatycki, "Differential structure of orbit spaces", Canad. J. Math. 53:4 (2001), 715-755. MR 2002j:53109 Zbl 01688933

[Godement 1973] R. Godement, Topologie algébrique et théorie des faisceaux, 3ème ed., Actualités scientifiques et industrielles 1252, Hermann, Paris, 1973. MR 49 \#9831 Zbl 0275.55010

[Kirwan 1985] F. C. Kirwan, "Partial desingularisations of quotients of nonsingular varieties and their Betti numbers", Ann. of Math. (2) 122:1 (1985), 41-85. MR 87a:14010 Zbl 0592.14011

[Koszul 1953] J. L. Koszul, "Sur certains groupes de transformations de Lie", pp. 137-141 in Géométrie différentielle (Strasbourg, 1953), Colloques Internationaux du CNRS, Centre National de la Recherche Scientifique, Paris, 1953. MR 15,600g Zbl 0101.16201

[Lerman and Tolman 2000] E. Lerman and S. Tolman, "Intersection cohomology of $S^{1}$ symplectic quotients and small resolutions", Duke Math. J. 103:1 (2000), 79-99. MR 2001k:53163 Zbl 0985. 53041

[Marsden and Weinstein 1974] J. Marsden and A. Weinstein, "Reduction of symplectic manifolds with symmetry”, Rep. Mathematical Phys. 5:1 (1974), 121-130. MR 53 \#6633 Zbl 0327.58005

[Sjamaar and Lerman 1991] R. Sjamaar and E. Lerman, "Stratified symplectic spaces and reduction”, Ann. of Math. (2) 134:2 (1991), 375-422. MR 92g:58036 Zbl 0759.58019

Received July 11, 2002.

\section{REYER SJAMAAR}

DEPARTMENT OF MATHEMATICS

CORNELL UNIVERSITY

ITHACA, NY 14853-4201

sjamaar@math.cornell.edu 